ting beim gleichnamigen Softwarehaus, zudem mit dem eBrief der PIN Mail AG. „Der eBrief verbindet moderne Technologie mit den Vorteilen der klassischen Kommunikation“, erläutert Krüger. „Der Behandler lädt seine Dokumente elektronisch hoch. Die Dokumente werden von der PIN Mail AG gedruckt, kuvertiert, frankiert und in die Zustellung übergeben.“ Alle Verbindungen für die Datenübertragung seien nach höchsten Sicherheitsstandards verschlüsselt und alle Mitarbeiter im Verarbeitungsprozess unterlägen sowohl dem Bundesdatenschutzgesetz als auch dem Postgeheimnis, versichert er.

\section{Patientenkommunikationskanäle}

Da die Kommunikation mit dem Patienten immer wichtiger wird, stehen auch hierfür Möglichkeiten bereit: Bei Epikur geht das per SMS-Versand aus dem System. Medatixx hat hierfür mit der Patientenservice-App „x.patient“ einen eigenen Kanal entwickelt, über den eine si- chere Verbindung zur Praxissoftware aufgebaut wird und dann direkt aus den Medatixx-Systemen heraus Daten mit Patienten ausgetauscht werden können. Natürlich lassen sich auch externe EMail-Dienste einbinden - die meisten Softwarehäuser können einen Zugriff etwa zu Microsofts Outlook oder Mozillas Thunderbird direkt über ihr System ermöglichen. Ob die Daten dann allerdings sicher übertragen werden, hängt am E-Mail-Provider. Indamed empfiehlt Praxen die sicherere TLS-Verschlüsselung zu nutzen, weil hier das Versandprotokoll in sechs Teile aufgesplittet werde. Um eine E-Mail lesen zu können, würden alle sechs Teile (für die Technikaffinen: Record-, Handshake-, Change-, Cipher-, Spec- und Alert-Protokoll) in der richtigen Reihenfolge benötigt, was potenzielle Angriffe erschwere. Die Verschlüsselung lässt sich laut Indamed dann ebenfalls direkt aus der Praxissoftware heraus anstoßen.

Rebekka Höhl

\title{
Health-Apps: Ein Siegel aus KV-Hand
}

\section{Die KV Telematik GmbH will ab 2018 zur Anlaufstelle im App-Dschungel werden - für Gründer, aber auch für Ärzte .}

$\mathrm{B}_{\mathrm{c}}^{\mathrm{a}}$ alsam für die Ärzte: Bremser in Sachen Digitalisierung sind die niedergelassenen Ärzte laut Dr. Florian Fuhrmann, Geschäftsführer der KV Telematik GmbH (KVTG), nicht. Ganz im Gegenteil: In den Praxen habe die Digitalisierung bereits vor über 30 Jahren mit der Einführung der Desktop-PC angefangen, erläuterte er auf dem gevkoSymposium „Digitales Gesundheitswesen 2021“ in Berlin. Genau diese frühe Digitalisierung im Gesundheitswesen werde nun zum Boomerang, denn die Transformation in die mobile Welt sei im Versorgungsalltag schwierig. Hier will die KVTG nun ansetzen, schließlich hätten die Praxen bis zu einer gewissen technischen Sättigung gut mitgemacht.

Ab 2018 will die KBV-Tochter Orientierung im undurchsichtigen HealthApp-Markt bieten. Dazu soll, wie Fuhrmann berichtete, ein systematisches Markt-Screening stattfinden. Gemein- sam mit medizinischen Fachgesellschaften wolle man ein inhaltliches Siegel für Health-Apps entwickeln. Gleichzeitig sieht sich die KVTG als Anlaufstelle für Unternehmen, Investoren und KVen, die neue Ideen in diesem Segment entwickeln wollen. Fuhrmann: „Wir wollen das Gesundheitswesen erläutern und Impulse setzen." Allerdings will die KVTG hier nicht als Geldgeber fungieren. „Wir wollen als Art Incubator ein Umfeld schaffen, das den Unternehmen hilft, sich im Gesundheitsmarkt zurechtzufinden."

Eher bekannt für Vernetzungsanwendungen wie den E-Arztbrief oder den Kommunikationskanal KV-Connect, will die KBV-Tochter Fuhrmann zufolge künftig aber auch eigene KV-Apps entwickeln. An erster Stelle steht dabei eine Notfall-App, die auch den Weg in die Telematikinfrastruktur finden soll.

RebekkaHöhl
Whitepaper hilft beim Umgang mit Cyberattacken

Cyberattacken mit Ransomware nehmen zu: Zirka 49\% der Unternehmen weltweit waren im Jahr 2016 mit mindestens einer Online-Erpressung konfrontiert. 39\% davon wurden mithilfe von Ransomware erpresst, das zeigt eine Studie der beiden IT-Dienstleister Dimension Data und Cisco Systems. Die Dienstleister haben nun ein Whitepaper veröffentlicht, das Betrieben beim richtigen Umgang mit Schadsoftware helfen soll, das auch für IT-affine Ärzte und Kliniken interessant sein dürfte. Kostenloser Download: http://bit.ly/2vGeg9Q

RebekkaHöhl

\section{medatixx: Schnellere Anbindung an das Labor}

Damit Praxen Laboraufträge elektronisch versenden können, hat der PraxisEDV-Anbieter medatixx nun die Order-Entry-Systeme "CyberLab", "LabUniq“, ,ixserv“, , AB+M“ sowie "QuickCon" an seine gleichnamige Praxissoftware angebunden. Hat das Labor den Auftrag erledigt, werden die Werte der Praxis ebenfalls elektronisch zurückgespielt, berichtet das Unternehmen. Dabei würden die Daten in strukturierter Form in das Laborblatt einfließen. Am Workflow ändert sich medatixx zufolge für die Praxen nichts. Ebenfalls neu ist, dass sich eine Kopie eines bereits erstellten Arztbriefes erzeugen lässt.

RebekkaHöhl

\section{EU: Sicherheitszertifikat für vernetzte Geräte}

Nach dem Willen der EU-Kommission soll es für Geräte, die sich mit dem Internet verbinden, künftig einheitliche europäische Sicherheitsstandards geben. Ähnlich wie bei Lebensmitteln könnten Verbraucher durch Sicherheitszertifikate erkennen, ob Produkte europäische Standards erfüllen, erklärte die Kommission. Die Bescheinigungen wären EU-weit gültig, aber nicht zwingend vorgeschrieben, hieß es. Sie sollen von der EU-Agentur für Cybersicherheit in Griechenland entwickelt werden, die den Plänen zufolge gestärkt werden soll. Dies bedarf aber noch der Zustimmung der EU-Staaten und des Europaparlaments. Derzeit sei es viel zu einfach, vernetzte Geräte anzugreifen, warnte der zuständige Vizepräsident der EU-Kommission, Andrus Ansip. 